\title{
FACTORES ASOCIADOS A SOMNOLENCIA DIURNA EN CONDUCTORES DE TRANSPORTE PÚBLICO DE LIMA METROPOLITANA
}

\author{
Karina Peña-Prado ${ }^{1, a}$, Jorge Rey de Castro ${ }^{2,3, b}$, Armando Talaverano-Ojeda ${ }^{4, c}$
}

\begin{abstract}
RESUMEN
Objetivos. Determinar la frecuencia de somnolencia y sus factores asociados en conductores de transporte público de Lima Metropolitana. Materiales y métodos. Estudio analítico transversal realizado en conductores de entre 18 y 65 años, que laboren en alguna empresa de transporte público registrada en la Municipalidad de Lima. La somnolencia se evaluó mediante una versión de la Escala de Somnolencia de Epworth validada en Perú. Las variables que se pudieron comportar como factores asociados se obtuvieron mediante una ficha de recolección de datos. Se utilizó la regresión logística para estimar la magnitud de la asociación entre la somnolencia y las variables consideradas como factores asociados. Resultados. Se incluyeron a 440 conductores, cuya mediana de edad fue 38,0 años y la mayoría $(99,3 \%)$ fueron varones. Del total de conductores el $17,7 \%$ (78) presentaron somnolencia diurna. Se encontró una asociación significativa entre la somnolencia y el sistema de rotación $2 \times 1(p=0,038)$ y entre la somnolencia y las horas de sueño menores a siete $(p=0,011)$. El análisis de regresión logística demostró que aquellos conductores con seis o menos horas de sueño tienen mayor probabilidad de somnolencia diurna (OR 1,83; IC95\%: 1,03-3,25). Conclusiones. Aproximadamente, uno de cada cinco conductores presentó somnolencia diurna, la cual estuvo asociada con tener seis o menos horas de sueño al día.
\end{abstract}

Palabras clave: Somnolencia; Trastornos de Somnolencia Excesiva; Sueño; Accidentes de Tránsito; Múltiples Morbilidades (fuente: DeCS BIREME).

\section{FACTORS ASSOCIATED WITH DAYTIME SLEEPINESS IN PUBLIC TRANSPORTATION DRIVERS IN LIMA METROPOLITAN AREA}

\begin{abstract}
Objective. To determine the frequency of sleepiness and its associated factors in public transportation drivers in Lima metropolitan area. Materials and Methods. Transversal analytical study carried out on drivers between 18 and 65 years old who work in a public transportation company registered in the Municipality of Lima. Sleepiness was assessed using a version of the Epworth Sleepiness Scale validated in Peru. The variables that could behave as associated factors were obtained by a data collection sheet. Logistic regression was used to estimate the magnitude of the association between sleepiness and variables considered as associated factors. Results. Four hundred forty (440) drivers were included, median age was 38.0 years and the majority (99.3\%) were males. From the total number of drivers, $17.7 \%(78)$ experienced daytime sleepiness. A significant association was found between sleepiness and the $2 \times 1$ rotation system $(p=0.038)$, and between sleepiness and hours of sleep under seven $(p=0.011)$. Logistic regression analysis showed that drivers with six or fewer hours of sleep were more likely to have daytime sleepiness (OR 1.83, 95\% Cl: 1.03-3.25). Conclusions. Approximately one out of five drivers experienced daytime sleepiness, which was associated with having six or fewer hours of sleep per day.
\end{abstract}

Keywords: Drowsiness; Excessive sleepiness disorders; Sleep; Traffic accidents; Multiple morbidities (source: MeSH NLM).

\section{INTRODUCCIÓN}

Los diversos sistemas de transporte público son de gran importancia para el funcionamiento de las grandes ciudades, ya que brindan servicios que la población requiere y utiliza de forma permanente. Además, facilita la movilización de las personas para el desarrollo de sus actividades diarias e influye en la dinámica social y económica de la sociedad ${ }^{(1)}$. Si bien, este sistema es de gran utilidad, también genera pérdidas por la gran cantidad de accidentes de tránsito que ocasiona, incluso se ha estimado que para el 2020 se convertiría en la principal causa de muerte ${ }^{(2)}$.

\footnotetext{
Unidad de Medicina Ocupacional y Medio Ambiente, Universidad Peruana Cayetano Heredia. Lima, Perú

Universidad Peruana Cayetano Heredia. Lima, Perú

Laboratorio del Sueño, Clínica Anglo Americana. Lima, Perú

Unidad de Medicina Ocupacional y Medio Ambiente. Universidad Peruana Cayetano Heredia. Lima, Perú

a Médico cirujano, maestro en Medicina Ocupacional y del Medio Ambiente; ${ }^{b}$ neumólogo; ${ }^{c}$ médico especialista en Medicina del Trabajo y Ergonomía, magister en Gerencia de Proyectos y Programas Sociales

El presente artículo forma parte de la tesis: Peña-Prado K. Somnolencia en conductores de transporte público regular de pasajeros de Lima Metropolitana - Perú. 2016 [Tesis de maestría en Internet]. Lima: Universidad Peruana Cayetano Heredia; 2017.

Recibido: 18/02/2019 Aprobado: 30/10/2019 En línea: 03/12/2019
}

Citar como: Peña-Prado K, Rey de Castro J, Talaverano-Ojeda A. Factores asociados a somnolencia diurna en conductores de transporte público de Lima Metropolitana. Rev Peru Med Exp Salud Publica. 2019;36(4):629-35. doi: 10.17843/rpmesp.2019.364.4305. 
Al respecto, en el 2015, la Organización Mundial de la Salud (OMS) manifestó que hubo 1,2 millones de fallecimientos por accidentes de tránsito ${ }^{(3)}$ y la Organización Panamericana de la Salud (OPS) declaró que dichas muertes ocurren principalmente en población joven, entre los 15 y 29 años ${ }^{(4)}$. Adicionalmente, se debe considerar que el $90 \%$ de las lesiones originadas se presentan en los países de bajos ingresos ${ }^{(2)}$.

Basándose en lo anterior, los trabajadores del transporte público deberían tener estilos de vida saludables, los cuales mejorarían su bienestar, y su capacidad funcional y laboral; dentro de estos estilos saludables se encuentra la óptima calidad de sueño que mantiene el buen funcionamiento de las áreas psicomotoras y neurocognitivas, además de mejorar el tiempo de reacción, vigilancia, juicio y atención del transportista, lo cual mejora la productividad del trabajador y la prevención de los accidentes ${ }^{(5,2)}$.

La somnolencia se vincula con las horas de labor al día, el tiempo de vigilia, la duración de la actividad laboral y la monotonía; y sus resultados que, por lo general son adversos, están relacionados con la seguridad. Por ejemplo, la somnolencia ha causado el $15 \%$ y $20 \%$ de accidentes de tránsito en China y Reino Unido, respectivamente, mientras que en Estados Unidos ha ocasionado el 40\% de los accidentes y más de 1500 muertes al año. Otros factores que podrían estar involucrados en la ocurrencia de accidentes de tránsito son la edad, el índice de masa corporal (IMC), la experiencia laboral, el tabaquismo, el alcohol, la agudeza visual, entre otros ${ }^{(6-10)}$.

En el Perú no se dispone de información relacionada a los conductores urbanos, quienes transportan a gran parte de la población, por tal motivo y en vista de la utilidad de los resultados, este estudio permite generar información epidemiológica de primera línea para esta actividad económica de riesgo, siendo promotor de la elaboración de futuros estudios bajo la misma línea de investigación, permitiendo la elaboración de guías o normas técnicas que sean de utilidad para actualizar y mejorar la normativa de la seguridad vial dirigida a las empresas de transporte público, conductores y peatones a nivel nacional.

Asimismo, propiciará que los directivos de las diversas empresas de transporte público tomen conciencia sobre la importancia de la salud de sus trabajadores en general y de su sistema de trabajo, pudiendo así brindar una mejor calidad de servicio y atención a toda la población. Por consiguiente, este estudio tiene como objetivo determinar los factores asociados a la somnolencia diurna en conductores de transporte público de buses en Lima Metropolitana en 2016.

\section{MATERIALES Y MÉTODOS}

El diseño del estudio fue transversal analítico. La población estuvo conformada por conductores del servicio de transporte público de buses de Lima Metropolitana. El número total

\section{MENSAJES CLAVE}

Motivación para realizar el estudio. Los sistemas de transporte público son de gran importancia y utilidad, porque facilitan la movilización de la población e influyen en la dinámica social y económica. Los conductores necesitan estilos de vida saludables para una eficiente labor

Principales hallazgos. Aproximadamente, uno de cada cinco conductores presentó somnolencia diurna. El sistema de rotación $2 \mathrm{x}$ 1 y tener seis o menos horas de sueño al día fueron los factores que se asociaron con la somnolencia diurna.

Implicancias. Este estudio contribuye con la identificación de los factores asociados a la somnolencia en conductores de transporte público de Lima Metropolitana.

de conductores fue proporcionado por la Gerencia de Transporte Urbano de la Municipalidad Metropolitana de Lima, que tenía registrados hasta setiembre de 2014 a 45410 conductores en la modalidad de transporte público regular de personas, dato que fue tomado como referencia para la elaboración del proyecto de investigación.

Se incluyeron conductores de 18 a 65 años, que trabajaban en alguna empresa de transporte público registrada en la Municipalidad de Lima y que aceptaron participar en el estudio mediante la firma del consentimiento informado y se excluyeron a conductores que, en el momento del estudio, no ejercían la actividad laboral. Para la selección de las empresas de transporte, se solicitó, mediante llamadas telefónicas, la autorización de sus representantes legales, contando finalmente con la autorización de seis de ellas.

La variable dependiente fue la somnolencia diurna, que fue medida a través de la versión peruana de la Escala de Somnolencia de Epworth (ESE-VP) ${ }^{(11)}$. El instrumento tiene ocho ítems y cada uno tiene un puntaje de 0 a 3 ( $0=$ nunca, 1=poca probabilidad de cabecear, 2=moderada probabilidad de cabecear, y $3=a l t a$ probabilidad de cabecear). El puntaje total de la escala está entre 0 y 24 . La forma de calcular el puntaje se consigue mediante la suma de los ocho ítems. Los puntajes mayores a 10 se considera como presencia de somnolencia diurna.

Las variables independientes fueron la edad, el sexo, el índice de masa corporal (IMC) (para medir el peso y la talla se utilizó instrumentos calibrados), el grado de instrucción, las horas de conducción por día (de naturaleza subjetiva), las horas de sueño por día (determinadas por la cantidad de horas utilizadas para dormir en un periodo de 24 horas, desde el momento en que el conductor se acuesta hasta el momento en que se levanta de la cama), antigüedad en el puesto de trabajo y el sistema de rotación (se identificaron dos sistemas de rotación: sistema $6 \times 1$, donde el conductor labora seis días consecutivos con uno de descanso y el sistema $2 \times 1$, donde el conductor labora dos días consecutivos y luego descansa un día, en ambos sistemas la actividad laboral se realiza de día y de noche). 
El cálculo de la muestra fue a través de la fórmula para población conocida, empleando el tamaño referencial de 45410 conductores, proporción de la población que tiene la característica de interés (50\%), el nivel de confianza $(95 \%)$ y el error de la estimación (5\%), obteniendo 381 casos. Además, se consideró una proporción esperada de pérdidas de $15 \%$, por lo que la muestra final fue de 449 conductores. El tipo de muestreo fue no probabilístico y la técnica de muestreo fue por conveniencia.

Entre febrero y abril del 2016 se coordinó fechas y horas para la recogida de información, ejecutándose entre dos a cinco días por cada mes, entre las 09:00 y 18:00 horas, en algunas ocasiones desde las 06:00 horas. Los paraderos donde se realizó la recolección de datos fueron los finales (lugar dónde se concentran los conductores para hacer cambio de turno o cambiar de unidad vehicular). Durante el trabajo de campo se garantizó la seguridad, tanto de los encuestadores como de los conductores, considerando que deberían estar en su descanso y en las instalaciones de las empresas.

Los encuestadores fueron dos estudiantes de psicología de sexto y séptimo ciclo, quienes se encontraban familiarizados con la herramienta (test de Epworth). Los encuestadores recibieron capacitaciones sobre los objetivos y variables del estudio, así como del correcto llenado de las fichas de recolección de datos. Asimismo, se realizó una prueba piloto a 85 conductores, antes del inicio del trabajo de campo. La autora corresponsal acompañó a los encuestadores durante el proceso de trabajo de campo.

Los datos obtenidos en las encuestas fueron codificados y se registraron en una base de datos en el programa Microsoft Excel 2013. Pasaron por una auditoría de calidad, para ello se contrató a dos digitadores quienes por separado pasaron la información al programa. Posteriormente, los autores contrastaron ambos archivos verificando la correspondencia de los datos ingresados.

El análisis estadístico se realizó aplicando el programa estadístico STATA versión 12. Las variables cualitativas, como la somnolencia diurna se reportaron a través de frecuencias y porcentajes, mientras que las variables cuantitativas se reportaron a través de la mediana y rangos intercuartílicos, ya que presentaron una distribución asimétrica. Para evaluar la relación entre las variables en estudio, se utilizó la prueba chi cuadrado de Pearson. Asimismo, se realizó el análisis de regresión logística donde la variable de resultado fue la somnolencia diurna y las variables predictoras fueron la edad mayor a 50 años, el grado de instrucción, el índice de masa corporal, el sistema de rotación y las horas de sueño. Se estimaron los valores de Odds Ratio (OR), así como sus intervalos de confianza al $95 \%$, siendo significativo un valor de $p<0,05$.

El estudio fue aprobado por el Comité Institucional de Ética para Humanos de la Universidad Peruana Cayetano
Heredia. Todos los participantes firmaron el consentimiento informado escrito de acuerdo con la Declaración de Helsinki.

\section{RESULTADOS}

Se incluyeron a 440 conductores de transporte público regular de Lima Metropolitana, con edades entre los 19 y 65 años, el 50,0\% fue mayor a 38 años, el 99,3\% fue de sexo masculino, un $43,0 \%$ presentó sobrepeso y el $74,5 \%$ tuvo secundaria completa. El 99,5\% de los conductores admitió conducir más de cinco horas por turno de trabajo, el $30,0 \%$ refiere que su sistema de rotación en la empresa es de $2 \times 1$, el $63,0 \%$ presentó entre tres y seis horas de sueño por día y el $32,5 \%$ de los conductores tenía entre 15 y 30 años de experiencia (Tabla 1).

Tabla 1. Características sociodemográficas de conductores de transporte público de Lima Metropolitana

\begin{tabular}{|c|c|}
\hline Características demográficas & n (\%) \\
\hline \multicolumn{2}{|l|}{ Sexo } \\
\hline Masculino & $437(99,3)$ \\
\hline Femenino & $3(0,7)$ \\
\hline Edad (años) * & $38(30,2-47,0)$ \\
\hline 19 a 29 & $92(20,9)$ \\
\hline 30 a 39 & $139(31,6)$ \\
\hline 40 a 49 & $112(25,5)$ \\
\hline 50 a más & $97(22,0)$ \\
\hline \multicolumn{2}{|l|}{ Grado de instrucción } \\
\hline Primaria completa o incompleta & $73(16,6)$ \\
\hline Secundaria completa & $328(74,5)$ \\
\hline Superior técnico o universitario & $39(8,9)$ \\
\hline Índice de masa corporal * & $28(25,4-31,2)$ \\
\hline Normal & $97(22,0)$ \\
\hline Sobrepeso & $189(43,0)$ \\
\hline Obesidad & $154(35,0)$ \\
\hline Horas de conducción * & $14(13-16)$ \\
\hline$\leq 5$ & $2(0,5)$ \\
\hline$>5$ & $438(99,5)$ \\
\hline \multicolumn{2}{|l|}{ Sistema de rotación } \\
\hline $2 \times 1$ & $132(30,0)$ \\
\hline $6 \times 1$ & $308(70,0)$ \\
\hline Horas de sueño por día * & $6(5-7)$ \\
\hline $3-6$ & $277(63,0)$ \\
\hline$>6$ & $163(37,0)$ \\
\hline Antigüedad en el puesto de trabajo (años) * & $20(5-12)$ \\
\hline$<5$ & $84(19,1)$ \\
\hline $5-10$ & $133(30,2)$ \\
\hline $10-15$ & $55(12,5)$ \\
\hline $15-30$ & $143(32,5)$ \\
\hline$>30$ & $25(5,7)$ \\
\hline \multicolumn{2}{|l|}{ Somnolencia diurna $^{\dagger}$} \\
\hline $\mathrm{Si}$ & $78(17,7)$ \\
\hline No & $362(82,3)$ \\
\hline
\end{tabular}


De acuerdo con la Escala de Somnolencia de Epworth, se catalogó como portadores de excesiva somnolencia diurna o presencia de somnolencia a 78 conductores $(17,7 \%)$ (Tabla 1). Asimismo, la somnolencia diurna se presentó en $31(23,5 \%)$ conductores del sistema de rotación $2 \times 1$ y en $47(15,3 \%)$ del sistema 6 x 1 .

Se evidenció una asociación estadísticamente significativa entre la presencia de somnolencia diurna y el sistema de rotación $2 \times 1$. Con relación a las horas de sueño por día, la somnolencia diurna se presentó en $59(21,3 \%)$ de los conductores con tres a seis horas de sueño y en $19(11,7 \%)$ de los que tenían más de seis horas, y esta asociación fue estadísticamente significativa (Figura 1).

El sistema de rotación de trabajo presentó asociación significativa con las horas de sueño por día de los conductores $(p=0,001)$, pero no con el tiempo de conducción $(p=0,353)$. Se evidenció que en los conductores que dormían entre tres y seis horas el $57,8 \%$ pertenecían al sistema $6 \times 1$ y en el $75 \%$ pertenecían al sistema $2 \times 1$. Por otro lado, la mayoría de los conductores de ambos sistemas de rotación (99,4\% y $100,0 \%$, respectivamente) refirió un tiempo de conducción mayor a cinco horas (Figura 2).

El análisis de regresión logística demostró que los conductores con seis o menos horas de sueño al día presentaron mayor probabilidad de somnolencia diurna (OR: 1,8; IC95\%: 1,03-3,25). Las otras variables incluidas en este análisis como la edad mayor a 50 años (OR: 1,36; IC95\%: 0,74-2,50), grado de instrucción secundaria (OR: 1,01; IC95\%: 0,51-1,98), grado de instrucción superior (OR: 0,34 IC95\%: 0,09-1,30), índice de masa corporal mayor a $25 \mathrm{~kg} / \mathrm{m}^{2}$ (OR: 1,35; IC95\%: 0,70-2,62) y el sistema de rotación de trabajo 2 x 1 (OR: 1,61; IC95\%: 0,95-2,74) no pudieron precisar con exactitud si existe mayor o menor probabilidad de somnolencia dado que estos resultados fueron no significativos ( $p>0,05)$ (Tabla 2).

\section{DISCUSIÓN}

Los conductores de transporte público de buses de Lima Metropolitana presentaron somnolencia durante la conducción, siendo las horas de sueño por día ( $\leq 6$ horas) el factor que se asocia de manera significativa con el desenlace. Adicionalmente, se pudo demostrar que los conductores, eran adultos varones, mayores de 38 años, tenían sobrepeso u obesidad, conducian por más de cinco horas en su turno de trabajo y dormían pocas horas.

Según la Escala de Somnolencia de Epworth, aproximadamente la quinta parte de los conductores presentaron somnolencia diurna, y de ellos más de la mitad laboraban en el sistema de rotación $2 \times 1$. Resultados similares fueron obtenidos en los estudios de Catarino et al. ${ }^{(12)}$ y de Guevara \& Torres ${ }^{(13)}$, quienes identificaron que menos de la cuarta parte de los conductores de taxis presentaban somnolencia excesiva diurna, mientras que resultados casi similares fueron hallados por Deza et al. ${ }^{(14)}$ quienes evidenciaron que la cuarta parte de los conductores de transporte interprovincial presentaron somnolencia diurna según la misma escala. Asimismo, Suarez Molina (15) y Caso et al. ${ }^{(16)}$ hallaron que más de la cuarta parte de los conductores presentaban somnolencia diurna.

Por otro lado, los estudios de Muñoz Escobar (17) y Santos et al. ${ }^{(18)}$ reportan resultados totalmente diferentes, considerando que el primero evidenció la presencia de

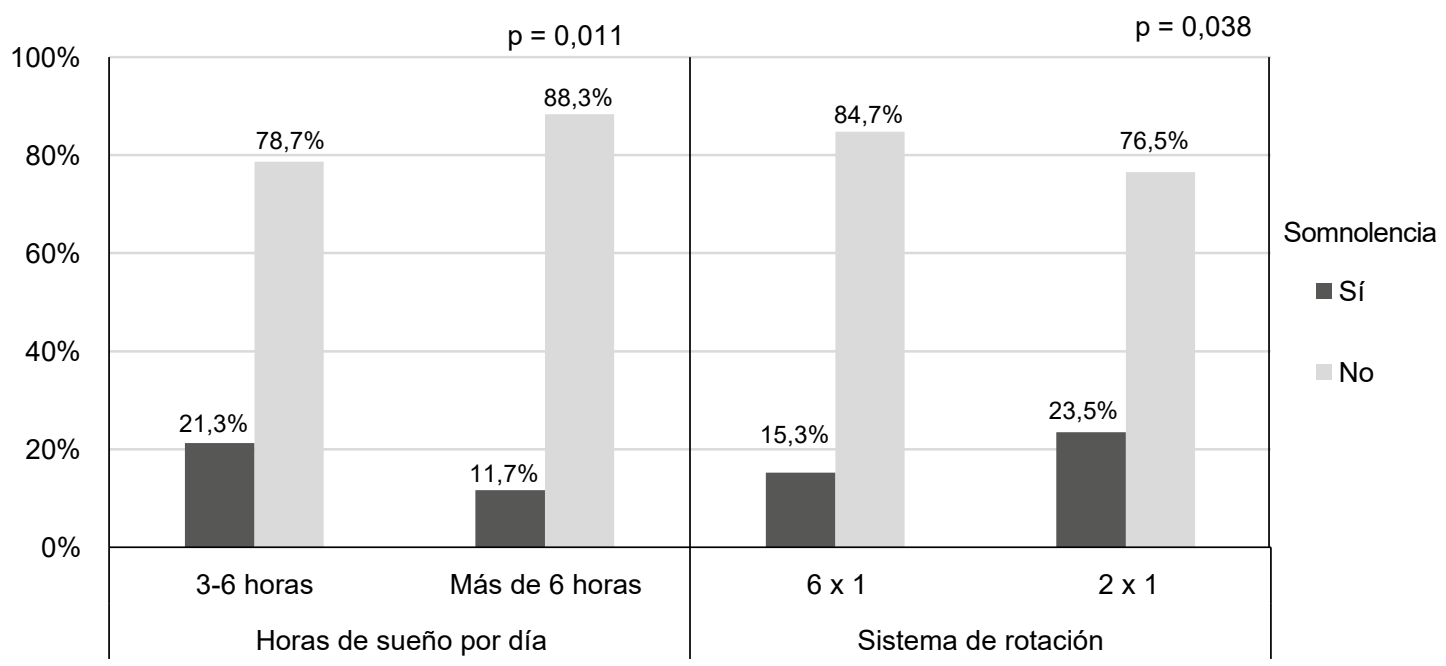

Figura 1. Horas de sueño por día y sistema de rotación según presencia de somnolencia en conductores de transporte público de Lima Metropolitana 


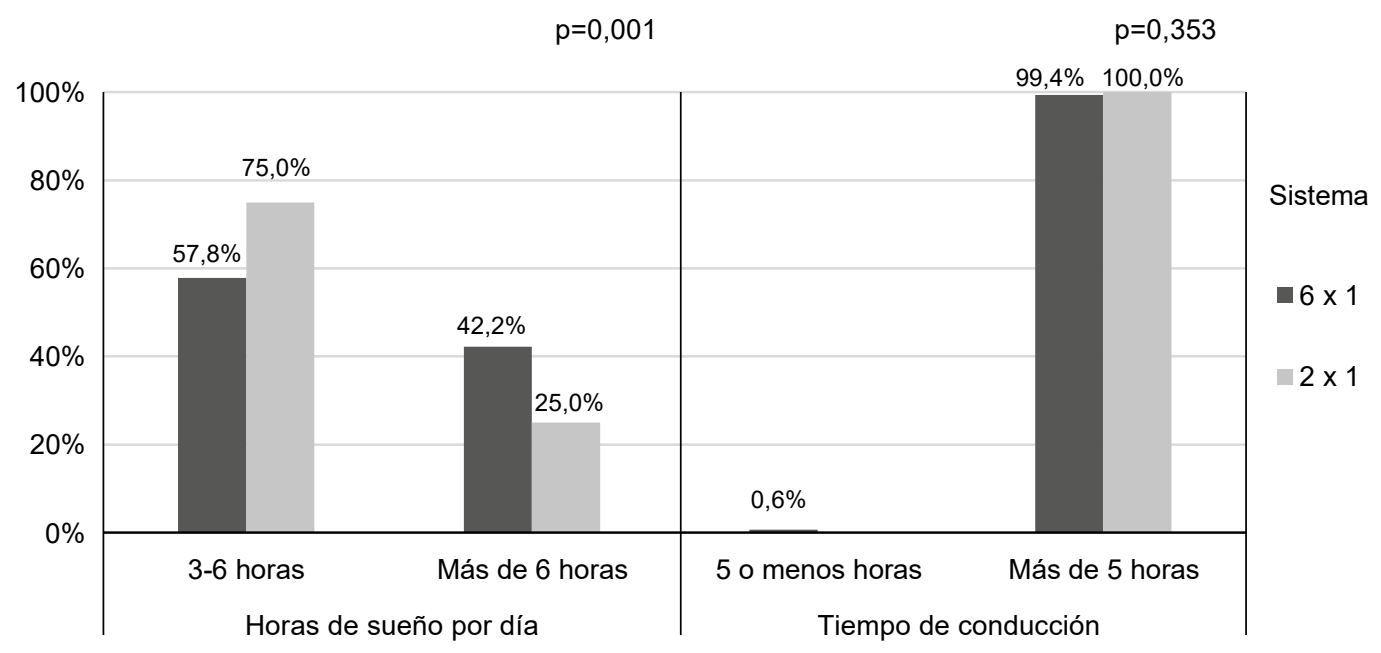

Figura 2. Horas de sueño por día y tiempo de conducción según sistema de rotación en conductores de transporte público de Lima Metropolitana

somnolencia excesiva en la mayoría de conductores de buses interurbanos, mientras que los segundos demostraron que la mayoría de los conductores de Brasilia tenían excesiva somnolencia diurna en comparación con los conductores de Florianópolis.

En general, al evaluar estudios semejantes, se puede evidenciar que los conductores de los diversos transportes públicos presentan un alto grado de somnolencia diurna, independientemente del horario laboral que puedan tener, esto podría deberse al alto grado de alerta y concentración que necesitan para la manipulación del vehículo, y de esta forma evitar los accidentes de tránsito.

Se evidenció que los conductores con mayor probabilidad de somnolencia eran los que tenían seis o menos horas de sueño por día. Al respecto, resultados similares fueron hallados por Suarez Molina ${ }^{(15)}$ quien evidenció que las horas de descanso y el peso de los conductores se asociaban de

Tabla 2. Factores asociados a la somnolencia diurna en conductores de transporte público de Lima Metropolitana

\begin{tabular}{lcccc}
\hline \multirow{2}{*}{ Variables } & \multirow{2}{*}{ ORa } & \multirow{2}{*}{$\begin{array}{c}\text { Valor } \\
\text { de } \mathbf{p}\end{array}$} & \multicolumn{2}{c}{ IC95\% } \\
\cline { 4 - 6 } & & Inferior & Superior \\
\hline Edad mayor a 50 años & 1,36 & 0,315 & 0,74 & 2,50 \\
Grado de instrucción & 1,01 & 0,986 & 0,51 & 1,98 \\
secundaria & 0,34 & 0,116 & 0,09 & 1,30 \\
$\begin{array}{l}\text { Grado de instrucción } \\
\text { superior }\end{array}$ & 1,35 & 0,368 & 0,70 & 2,62 \\
Índice de masa corporal & 1,62 & 0,074 & 0,95 & 2,74 \\
$>25 \mathrm{~kg} / \mathrm{m}^{2}$ & 1,83 & 0,040 & 1,03 & 3,25 \\
\hline Sistema de rotación 2 x 1 & & &
\end{tabular}

ORa: Odds ratio ajustado, IC95\%: intervalo de confianza al 95\% Modelo de regresión logística ajustado a edad, grado de instrucción, índice de masa corporal, sistema de rotación y las horas de sueño. manera significativa con la somnolencia diurna. Asimismo, el estudio de Guevara \& Torres ${ }^{(13)}$ destacó como factores asociados a los conductores jóvenes y al menor grado de instrucción. Mientras que Intriago Alvarez ${ }^{(19)}$ presentó resultados diferentes, donde las horas de sueño de los conductores de buses interprovinciales no se asociaron a la somnolencia. Este factor resultaría ser modificable, ya que si se mejorarían los horarios laborales, se podría incrementar directamente las horas de sueño en los conductores de transporte público.

Las características sociodemográficas de los participantes de los diversos estudios relacionados con la presente investigación no pueden ser contrastadas, debido a que cada una de las características poblacionales en análisis son propias, solo se mencionarán las características más resaltantes halladas en cada uno de los estudios, por ejemplo, los estudios de Anund et al. (20), Muñoz Escobar (17), Deza et al. (14), Intriago Alvarez ${ }^{(19)}$ y Suarez Molina ${ }^{(15)}$ concuerdan en que la mayoría de los conductores que participaron en cada uno de los estudios presentaban sobrepeso u obesidad. Al respecto, se puede mencionar que estos problemas nutricionales, podría deberse al sedentarismo que los conductores experimentan durante toda su actividad laboral, sin opción de realizar algún tipo de ejercicio físico, puesto que esta actividad demanda de una gran concentración por parte del conductor.

Por otro lado, Intriago Alvarez ${ }^{(19)}$ obtuvo resultados casi semejantes con el presente estudio, ya que los conductores tenían un promedio de 19 años de experiencia laboral y dormían menos de seis horas diarias, mientras que Muñoz Escobar ${ }^{(17)}$ identificó que los conductores por lo general tenían una experiencia laboral entre tres a diez años y dormían entre siete a ocho horas diarias. Estos resultados demuestran que no existe un consenso respecto a las 
horas de sueño en los conductores, independientemente del sistema de rotación al cual puedan estar sujetos en su jornada laboral, lo recomendable para cualquier persona es que el periodo de sueño sea entre siete y ocho horas diarias ${ }^{(21)}$, para poder realizar las actividades de manera eficiente, aplicándose esto también a los conductores.

Sobre las horas de conducción, diferentes estudios muestran resultados semejantes, donde el tiempo de conducción por lo general es mayor de 10 horas ${ }^{(14,15)}$, esto demuestra que la actividad laboral de los conductores es inversamente proporcional a sus horas de sueño, pudiendo ser este el motivo por el cual los conductores presenten excesiva somnolencia diurna; además, en el presente estudio la somnolencia diurna se podría atribuir al sistema de rotación, teniendo en consideración el turno laboral de cada uno de los conductores, como es el caso del estudio de Gastaldi et al. ${ }^{(22)}$.

Adicionalmente, respecto a las horas de jornada laboral, los resultados revelan que los conductores laboran más de cinco horas, independientemente del turno y del sistema de trabajo. Sin embargo, el Decreto Supremo № 025-2017MTC, estipula que los conductores de transporte público no deben realizar su actividad durante más de cinco horas continuas cuando se trata de un turno diurno o no más de cuatro horas continuas cuando se trata de un turno nocturno; además, las horas acumuladas de conducción no deben de ser de más de 10 horas en un lapso de 24 horas, contando desde la hora en que se inicia la prestación del servicio ${ }^{(23)}$

Ambos aspectos señalan que los conductores tienen su salud afectada, no sólo porque la mayoría tiene sobrepeso u obesidad, sino porque la falta de sueño y descanso debilita su sistema inmunitario, provocando una mayor frecuencia de patologías virales, lo cual repercute, no sólo de forma directa en la salud del conductor, sino de forma indirecta en el aspecto económico, laboral y social, tanto del propio trabajador como de la empresa.

Como limitaciones del estudio se debe mencionar el retraso en el otorgamiento de las autorizaciones brindadas por las empresas de transporte público lo que dificultó el acceso a los conductores; el muestreo no probabilístico que no permite que estos hallazgos se puedan inferir a toda la población de conductores de Lima Metropolitana y el desconocimiento de la ubicación de cada conductor por parte de la empresa, lo que limitaba el acceso. Además, es importante mencionar que la Escala de Somnolencia de Epworth brinda datos subjetivos, ya que es muy probable que los conductores oculten información para proteger su puesto laboral, esto supondría que la proporción de conductores con somnolencia sea mayor a la encontrada, sin embargo, por las facilidades de aplicación que brinda este instrumento se vio por conveniente su uso.

En conclusión, se pudo evidenciar que aproximadamente una quinta parte de los conductores evaluados presentaban somnolencia diurna durante su labor diaria y los factores asociados fueron el sistema de rotación $2 \times 1$ y tener seis o menos horas de sueño al día. Aunque el IMC no fue un factor asociado a la somnolencia, se debe mencionar que es una característica de importancia en esta población, ya que podría estar relacionada con otras patologías concomitantes. Es importante la revisión de las normas legales vigentes y de la intervención de los órganos fiscalizadores correspondientes. Asimismo, se debería implementar capacitaciones relacionadas con la higiene, calidad de sueño y factores asociados, dirigido no sólo a los conductores, sino también a los directivos de las empresas de transporte público, identificando la importancia de la estandarización de los descansos, los sistemas de rotación y el cumplimiento de las horas de sueño recomendadas para el ser humano. Ello influiría en la reducción de la incidencia de los accidentes de tránsito, mejorando de manera directa en la salud pública nacional.

Contribuciones de autoría: KPP, JRC y ATO han participado en la concepción del artículo, la recolección de datos, su redacción y aprobación de la versión final.

\section{Fuentes de financiamiento: autofinanciado}

Conflictos de interés: se declara que no existen conflictos de interés para la publicación del artículo.

\section{REFERENCIAS BIBLIOGRÁFICAS}

1. Oviedo-Oviedo NV, Sacanambuy-Cabrera JM, Matabanchoy-Tulcan SM, ZambranoGuerrero CA. Percepción de conductores de transporte urbano, sobre calidad de vida laboral. Rev Univ Salud. 2016;18(3):43246. doi: $10.22267 /$ rus.161803.49.

2. RiscoJ, RuizP,Mariños A,Juarez A, Ramos M, Salmavides F, et al. Excessive Sleepiness Prevalence in Public Transportation Drivers of a Developing Country. Traffic
Inj Prev. 2013;14(2):145-9. doi: 10.1080 / 15389588.2012 .692493$.

3. Organización Mundial de la Salud. Informe sobre la situacion mundial de la seguridad vial 2015. [Internet]. Ginebra:OMS; 2015 [citado 23 septiembre 2019]. Disponible en: https://www. who.int/violence_injury_prevention/ road_safety_status/2015/es/
4. Organización Panamericana de la Salud. La Seguridad vial en las región de las Américas [Internet]. Washington, DC: OPS, 2016 [citado 23 septiembre 2019]. Disponible en: http://iris.paho.org/ xmlui/handle/123456789/28565

5. GomeroCuadra R.Experienciaenlagestión de la somnolencia de conductores mineros peruanos a gran altitud, 2008-2014. Rev Asoc Esp Med Trab. 2017;(26):137-45. 
6. Phatrabuddha N, Yingratanasuk T, Rotwannasin P, Jaidee W, Krajaiklang N. Assessment of Sleep Deprivation and Fatigue Among Chemical Transportation Drivers in Chonburi, Thailand. Saf Health Work. 2018;9(2):159-63. doi: 10.1016/j. shaw.2017.06.014.

7. Zhang G, Yau K, Zhang X, Li Y. Traffic accidents involving fatigue driving and their extent of casualties. Accid Anal Prev. 2016;87:34-42. doi: 10.1016/j. aap.2015.10.033.

8. Bon T,Santos C, Mateus C, areal A, Trigoso J, Nunes C. Road traffic accidents and selfreported Portuguese car driver's attitudes, behaviors, and opinions: Are they related? Traffic Inj Prev. 2016;17(7):705-11. doi: $10.1080 / 15389588.2016 .1150591$.

9. Stevenson M, Elkington J, Sharwood L, Meuleners L, Ivers R, Boufous S, et al. The role of sleepiness, sleep disorders, and the work environment on heavyvehicle crashes in 2 Australian states. Am J Epidemiol. 2014;179(5):594-601. doi: 10.1093/aje/kwt305.

10. Ebrahimi M, Sadeghi M, Dehghani M,Niiat $K$. Sleep habits and road traffic accident risk for Iranian occupational drivers. Int J Occup Med Environ Health. 2015;28(2):305-12. doi: 10.13075/ijomeh.1896.00360.

11. Rosales E, Rey de Castro J, Huayanay L, Zagaceta K. Validation and modification of the Epworth Sleepiness Scale in Peruvian propularion. Sleep Breath. 2012;16(1):5969. doi: 10.1007/s11325-011-0485-1.

12. Catarino R, Spratley J, Catarino I, Lunet N, Pais M. Sleepiness and sleepdisordered breathing in truck drivers : risk analysis of road accidents. Sleep Breath. 2014;18(1):59-68. doi: 10.1007/s11325013-0848-x.

13. Guevara Ipushima KG, Torres Jara ET. Calidad de sueño y somnolencia diurna en conductores de taxi de una empresa privada en Lima Metropolitana-Santiago de Surco, 2016 [Tesis en Internet]. Lima: Universidad Privada Cayetano Heredia; 2016 [citado 23 septiembre 2019]. Disponible en: http://repositorio.upch. edu.pe/handle/upch/647

14. Deza F, Rey de Castro J, Gonzales C, León F, Osada J, Rosales E. Sleep habits, fatigue, and sleepiness in Chiclayo-Peru's bus drivers. Sleep Breath. 2017;21(3):745-749. doi: 10.1007/s11325-017-1502-9.

15. Suárez Molina A. Somnolencia diurna y factores asociados en conductores de transporte público que asistieron a un centro de reconocimiento de conductores en la ciudad de Cali. 2018-19 [Tesis de maestría en Internet]. Cali: Universidad del Valle; 2019. [citado 20 enero 2019]. Disponible en: http://bibliotecadigital.univalle.edu. co/bitstream/10893/13781/2/CB0592576.pdf

16. Caso A, Rey de Castro J, Rosales-Mayor E. Hábitos del sueño y accidentes de tránsito en conductores de ómnibus interprovincial de Arequipa, Perú. Rev Peru Med Exp Salud Publica. 2014;31(4):707-11.

17. Muñoz Escobar FD. Fatiga, somnolencia y accidentabilidad en conductores de buses interurbanos [Tesis en Internet]. Concepción: Universidad de Concepción; 2018 [citado 20 enero 2019]. Disponible en: http://repositorio.udec.cl/handle/11594/3053

18. Santos DB, Bittencourt LG, Viegas CA, Gaio E. Daytime sleepiness and attention in city bus drivers of two capitals of Brazil. Rev Port Pneumol. 2013;19(4):152-6. doi: 10.1016/j. rppneu.2013.01.001.

19. Intriago Alvarez MB. Relación del síndrome de apnea obstructiva del sueño con accidentes de tránsito en conductores profesioanles de buses intercantonales e interprovinciales del Guayas, AgostoOctubre 2016 [Tesis en Internet]. Guayas: Universidad de Especialidades Espíritu Santo; 2017 [citado 20 enero 2019]. Disponible en: http://repositorio.uees. edu.ec/handle/123456789/2690

20. Anund A, Ihlstrom J, Fors C, Kecklund G, Filtness A. Factors associated with self-reported driver sleepiness and incidents in city bus drivers. Ind Health. 2016;54(4):337-46. doi: 10.2486/indhealth.2015-0217.

21. Hirshlowitz M, Whiton K, Albert S, Alessi C, Bruni O, Doncarlos L, et al. National Sleep Foundation's sleep time duration recommendations: methodology and results summary. Sleep Health. 2015;1(1):40-43. doi: 10.1016/j.sleh.2014.12.010.

22. Gastaldi M, Rossi R, Gecchele G. Effects of driver task-related fatigue on driving performance. Procedia Soc Behav Sci. 2014;(111):955-64. doi: 10.1016/j. sbspro.2014.01.130.

23. Decreto Supremo que modifica el Reglamento Nacional de Administracion de Transporte, aprobado por Decreto Supremo No17-2009-MTC y establece otras disposiciones en materia de transporte y tránsito terrestre. Decreto Supremo $\mathrm{N}^{\circ}$ 025-2017-MTC. 2017. El Peruano p. 46 (28-12-2017). Disponible en: https:// busquedas.elperuano.pe/normaslegales/ decreto-supremo-que-modifica-el-reglamento-nacional-de-admin-decreto-supremo-n-025-2017-mtc-1601502-7/

Correspondencia: Karina Peña Prado

Dirección: Av. 6 de agosto 768. Jesús María. Lima, Perú

Teléfono: (+51) 941380384

Correo electrónico: karina.pena@upch.pe 